\title{
Real-Time Gigabit-Ethernet Transmission over Optical Wireless Using Off-the-Shelf Components
}

\author{
G. Cossu(1) W. Ali(1),A. Sturniolo(1), A. Messa(1), E. Ciaramella(1)
}

(1) Scuola Superiore Sant'Anna - TeCIP, via G. Moruzzi 1, 56124, Pisa, Italy, g.cossu@santannapisa.it

\begin{abstract}
We experimentally demonstrate real-time Gigabit-Ethernet optical wireless transmission exploiting commercially available components. The system does not require amplification or emphasis stages and works at $2 \mathrm{~m}$ distance with good robustness to misalignment $(>10 \mathrm{~cm})$.
\end{abstract}

\section{Introduction}

The well-known trend of growing indoor data traffic and high data rate requirements demand novel wireless solutions to meet these challenges. Millimetre waves transmission has been explored to provide Gigabit capacity for indoor data transmission ${ }^{1}$, but these frequencies require high-speed amplifiers and mixers, not widely available ${ }^{2}$. Recently, optical wireless communication (OWC) is considered a strong candidate for high-speed indoor wireless transmission.

As a complementary approach to RF systems, OWC can reach much higher bit-rates ${ }^{3}$. Among OWC solutions, those based on visible Light Emitting Diodes (LEDs) have already demonstrated Gigabit-class connectivity ${ }^{4}$. However, reaching these speeds by LEDs requires OFDM to fully exploit the small available modulation bandwidth of the devices and can only be achieved by employing digital/analog signal processing.

Unlike LEDs, visible Vertical Cavity Surface Emitting Lasers (VCSELs) exhibit larger bandwidth and provide Gigabit data rates with no need of complex signal processing. First feasibility of Gigabit Ethernet OWC based on VCSELs was proposed by ${ }^{5}$, using three high power VCSELS and three Avalanche PhotoDiodes (APDs). However, that configuration resulted in high complexity, high power consumption and additional cost.

Here, we report a real-time Gigabit Ethernet OWC system based on a single VCSEL and
APD. We demonstrated a transmission distance of $2 \mathrm{~m}$ with a very high robustness to receiver misalignment $(>10 \mathrm{~cm})$ considering a BER target of $10^{-9}$. This is confirmed by Bit Error Ratio (BER) measurements at different positions and at different received optical power. We used the OWC system to realize an Ethernet connection and then to transmit a streaming HD video between two PCs without packet-loss. The proposed solution provides a wireless system fully compliant with the Gigabit Ethernet standard.

\section{Experimental details}

The experimental setup of the optical wireless system is represented in Fig. 1. The transmitter (TX) was simply made of a commercially available VCSEL (emission at $680 \mathrm{~nm}, 5 \mathrm{GHz} 3$ $\mathrm{dB}$ bandwidth, $2.2 \mathrm{~mW}$ maximum output power and $20^{\circ}$ beam divergence). For the measurements, we fixed the bias current at 4.6 $\mathrm{mA}$, hence the average output power was $1.7 \mathrm{~mW}$. A 1 -inch bi-convex lens ( $F_{1}=1$ inch) was placed in front of the TX to reduce the beam divergence down to around $2^{\circ}$. The TX was placed on a 3-axis translator stage to precisely align the VCSEL with the TX lens.

The link distance was fixed at $z=2 \mathrm{~m}$, which is average ceiling-desktop distance in indoor. The power density at $z$ was measured to be around $1.5 \mathrm{~mW} / \mathrm{m}^{2}(1-2 \mathrm{~lx})$, quite lower than the eye safety standards ${ }^{6}$.

The receiver (RX) was made by an APD $(0.5 \mathrm{~mm}$ diameter active area, $1 \mathrm{GHz} 3-\mathrm{dB}$ bandwidth,

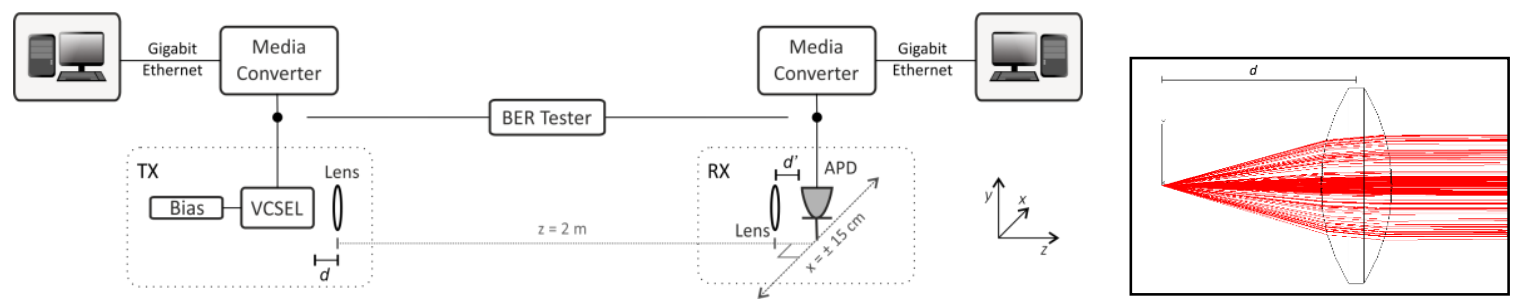

Fig. 1: Experimental setup for video transmission and BER measurements. VCSEL: Vertical Cavity Surface Emitting LASER. APD: Avalanche PhotoDiode. Switches indicate that Signal Quality Analyzer and Media Converters were alternatively used. In the inset, the ray-tracing simulation of the VCSEL and the used optical lens 
responsivity at $680 \mathrm{~nm}$ of $38 \mathrm{~A} / \mathrm{W})$. Another biconvex lens $\left(F_{2}=1 \mathrm{inch}\right)$ was placed in front of the APD to focus properly the light into the active area; the APD was set at the focal plane of the lens. To simplify the tolerance measurements, the $\mathrm{RX}$ module was placed on an optical rail along the $\mathrm{X}$-axis. The output of the APD was connected to the BER analyser. The same signal was also monitored on the sampling oscilloscope for eye diagram analysis.

Two sets of measurements were performed. First, we characterized the system performance: the VCSEL and the APD were connected to a BER-Tester (BERT), which generated a 1.25 Gb/s NRZ (PRBS sequence, $2^{7}-1$ long) and measured the $B E R$ at $R X$. The signal amplitude from the BERT was fixed at $560 \mathrm{mV}$, which corresponds to the amplitude from common Ethernet cards (the exact value is taken from the media converter defined below). To make the system simpler, no amplifier was used to increase the modulation index, which was presently around 0.3 . For the video transmission using the Ethernet interfaces, the electrical output of an Ethernet card of PC-1 was converted into a 1000BASE-SX Ethernet standard, a $1.25 \mathrm{~Gb} / \mathrm{s}$ NRZ signal by a standard Media Converter (MC). The VCSEL was then directly modulated by the output of the MC. After propagation, the signal was detected by the $R X$ and the electrical signal was then sent to another MC whose output was eventually sent to the Ethernet interface of a second PC.

\section{Numerical design}

First, we performed a simulation using a raytracing software to optimize the system parameters. We defined the TX and RX based on the VCSEL and APD above parameters. We defined the distance between TX source and lens as $d$ (as reported in inset of the Fig. 1). We estimated the received optical power along the $X$-axis considering different values of $d$ for fixed configuration of the RX, i.e., fixed distance $d$ ' $\left(d^{\prime}=F_{2}\right)$ between the RX lens and the APD and RX alignment parallel to the optic axis.

For any $d$ value, the simulation was realized moving the TX bi-convex lens along the Z-axis in order to maximize the beam spot at $2 \mathrm{~m}$ distance, while keeping enough optical power to reach a good BER (-40 dBm, in our case).

The results are presented in Fig. 2. From this simulation, we see that, up to around $6 \mathrm{~cm}$ displacement, the power levels exceed the sensitivity (sensitivity is indicated by a grey line in
Fig. 2). This can be achieved in the configuration where $d$ is around $18 \mathrm{~mm}$. We report the calculated beam profile at $2 \mathrm{~m}$ distance in Fig. 3 . The simulations assisted to design the OWC system with minimum use of optics at TX and RX by achieving the higher tolerance range.

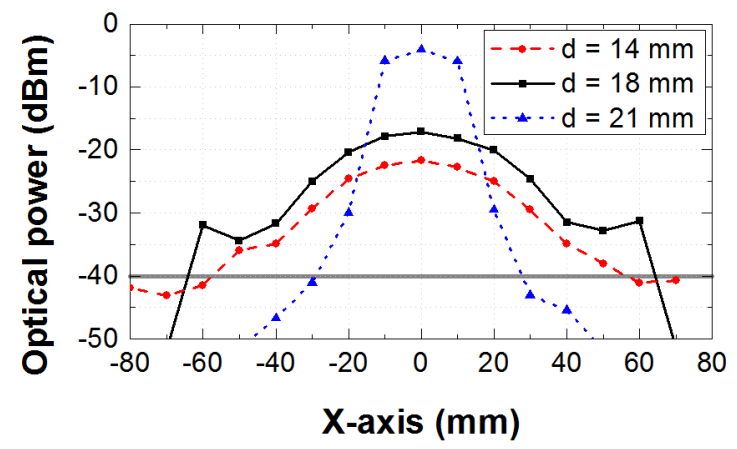

Fig. 2: Ray-tracing simulation of received optical power as a function of the receiver position on X-axis. The curves are taken for different values of $d$ (distance between TX source and lens).

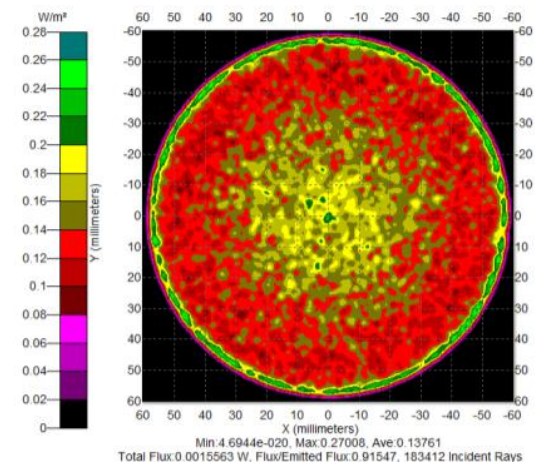

Fig. 3: Irradiance map of the incident flux at $2 \mathrm{~m}$ distance

\section{$1.25 \mathrm{~Gb} / \mathrm{s}$ transmission}

We first characterized the OW RX. Figure 4 reports the BER values as a function of the received optical power. From this plot, we see that a received optical power of $-40 \mathrm{dBm}$ is the minimum to achieve a BER lower than $10^{-9}(\mathrm{RX}$ sensitivity).

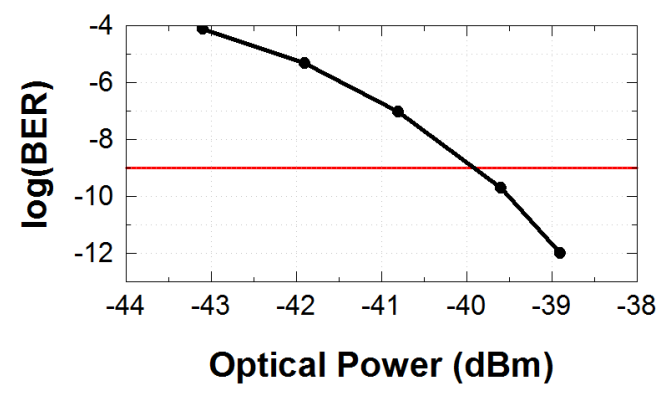

Fig. 4: BER measurements vs. the received optical power. The straight line at -9 indicates the target BER.

We then measured the tolerance to misalignment: the $R X$ module (APD and lens) was moved along the $\mathrm{X}$-axis orthogonal to the $\mathrm{Z}$ - 
axis, as shown in Fig. 1. We assume that the system tolerance is the spatial range where BER is below $10^{-9}$.

$\mathrm{TX}$ and $\mathrm{RX}$ were aligned by maximizing the eye opening. The bias current of the VCSEL was also optimized in order to maximize the signal quality. Fig. 5 depicts the wide-open eye diagram of $1.25 \mathrm{~Gb} / \mathrm{s}$ stream in the aligned condition $(X=0 \mathrm{~cm})$.

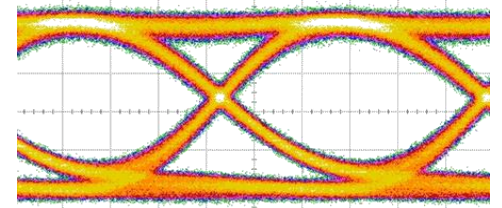

Fig. 5: Eye diagram in aligned position at $2 \mathrm{~m}$ distance. Scale: $150 \mathrm{ps} / \mathrm{div} ; 65 \mathrm{mV} / \mathrm{div}$

In this configuration, we experimentally estimated a BER value lower than $10^{-12}$ (no error after 30 minutes). After that, we continuously shifted the $\mathrm{RX}$ along the $\mathrm{X}$-axis until BER became higher than $10^{-6}$. These observations are summarized in Fig. 6. For a wide range $( \pm 6 \mathrm{~cm})$, the BER was lower than $10^{-12}$. Beyond this range, the BER increased abruptly in few millimetres (in both \pm directions). This was because the beam spot generated after the TX lens had a profile quite sharp with neat edges.

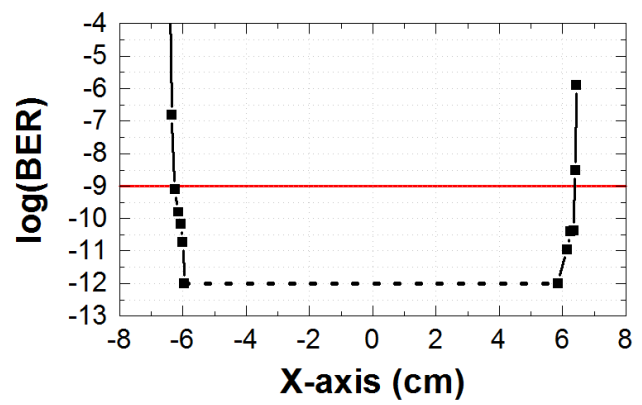

Fig. 6: BER measurements as a function of the receiver position on $\mathrm{X}$-axis. The dashed line indicates that the measured BER is lower than $10^{-12}$; the straight line at $10^{-9}$ indicates the target BER

Considering a target BER of $10^{-9}$, the tolerance range was around $12 \mathrm{~cm}$, i.e., the system can cover an area up to of $120 \mathrm{~cm}^{2}$ at $2 \mathrm{~m}$ distance. The eye diagram at BER $=10^{-9}$ is also reported in Fig. 7.

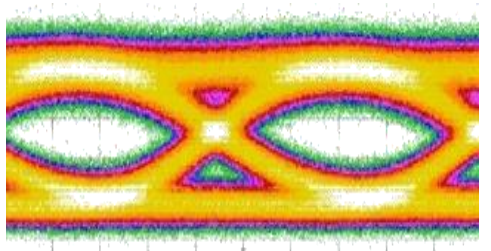

Fig. 7: Eye diagram at the edge of the spot $\left(B E R=10^{-9}\right)$. Scale: $150 \mathrm{ps} / \mathrm{div}$; $8 \mathrm{mV} / \mathrm{div}$
A data transmission was finally performed exploiting a freeware software able to generate and receive the data through the Gigabit Ethernet common card interfaces: the packets were generated on PC-1 and transmitted to PC-2 using OWC link. On PC-2 the software detected the received data and measured any packet loss. As expected, in aligned position, zero packet loss was recorded. HD video transmission between two PCs was also successfully demonstrated. We tested both TCP and UDP protocols, in both cases zero packet loss was found.

\section{Conclusion}

We experimentally demonstrated a simple optical wireless transmission compliant with the GigabitEthernet standard. The designed system operated with power efficient components and few optics. The performance were first simulated with a ray-tracing software and then experimentally proven. BER measurements were taken at different positions to estimate a tolerance to misalignment range. The tolerance was found to be higher than $10 \mathrm{~cm}$, providing $120 \mathrm{~cm}^{2}$ area with $\mathrm{BER}<10^{-9}$. The operation was also demonstrated with a video transmission. System performance can be further improved by adding an electrical amplifier at TX and at RX, although these could increase the hardware complexity.

\section{Acknowledgements}

This work has been partly supported by PORFESR project FIPILI3 funded by the Tuscany Regional Government.

\section{References}

[1] Rappaport, Theodore S., et al. "Millimeter wave mobile communications for $5 \mathrm{G}$ cellular: It will work!." IEEE access 1 (2013): 335-349.

[2] Gomez, Ariel, et al. "Beyond 100-Gb/s indoor wide fieldof-view optical wireless communications." IEEE Photon. Technol. Lett. 27.4 (2015): 367-370.

[3] Wang, Yiguang, et al. "8-Gb/s RGBY LED-based WDM VLC system employing high-order CAP modulation and hybrid post equalizer." IEEE Photonics Journal 7.6 (2015): 1-7.

[4] Cossu, G., et al. "Gigabit-class optical wireless communication system at indoor distances (1.5-4 m)." Optics express 23.12 (2015): 15700-15705.

[5] Minh, H. L., et al. "Bidirectional gigabit ethernet optical wireless communications system for home access networks." IET communications 6.11 (2012): 1529-1536.

[6] IEC 60825-1 Ed. 3.0 (2014); Safety of laser products Part 1: Equipment classification and requirements 